\title{
SOLITARY EOSINOPHILIC GRANULOMA OF BONE
}

\author{
T. HUNTER, ROMford, ENGLAND \\ From the Oldchurch Hospital, Romford
}

Since eosinophilic granuloma first achieved the status of a pathological and clinical entity in 1940, through the simultaneous publications of Jaffe and Lichtenstein, and of Otani and Ehrlich, a considerable number of papers, mainly by American authors, have appeared on the subject. The histopathology has been thoroughly investigated and described by Green and Farber (1942), Jaffe and Lichtenstein (1944), Ponseti (1948) and others. Its relationship to Hand-Schüller-Christian disease and to Letterer-Siwe disease appears to have been satisfactorily established. Ponseti (1948) concluded that these conditions represent different clinical and anatomical manifestations of the same underlying pathological process, of which the basic feature is reticular hyperplasia. The solitary eosinophilic granuloma of bone is the mildest clinical form of this group of diseases.

Although eosinophilic granuloma of bone may also occur as multiple lesions, Hill (1949), in a comprehensive review of the literature, estimated that in 71.2 per cent of cases it is manifest as a solitary focus, and it is with the latter type that this paper deals. Eight cases are described. Three of those have been encountered in one hospital during the past year, indicating that the lesion is probably not one of excessive rarity. It is thought worthwhile, therefore, to illustrate its radiographic appearances, which are of particular interest in relation to differential diagnosis, and to discuss briefly its pathology, clinical features, and treatment. Two possible complications are also described.

\section{CASE REPORTS}

Case 1-A girl aged five years complained of slight pain in her right shoulder, appearing after a fall two weeks before she attended hospital. On examination, the lateral part of the right clavicle was found to be thickened and slightly tender. Clinical examination was otherwise negative.

Radiographs (Fig. 1) showed an area of destruction in the lateral half of the right clavicle, partly involving the cortex, and a considerable amount of surrounding subperiosteal new bone formation. A fine crack fracture through the bone at the point of maximum destruction was just discernible.

Investigations - The serum alkaline phosphatase was slightly raised (21.0 KA units per cubic millimetre) and there was a leucocytosis $(16,200$ per cubic millimetre) with a normal differential count. The remaining routine laboratory tests were normal.

A biopsy excision was done. The pre-operative diagnosis rested between a sarcoma, an eosinophilic granuloma, and a low-grade pyogenic lesion.

Operation-The outer half of the right clavicle, including the whole of the diseased area, was excised. Examination showed that the affected part of the bone contained soft yellowish material replacing the normal cancellous tissue.

Histology-The appearances of this material were those of an eosinophilic granuloma. Culture was sterile except for a scanty growth of staphylococcus albus.

Progress-The patient's post-operative course was uneventful. The operation wound healed by first intention and two weeks later she was discharged from hospital, having rapidly regained a full, painless range of movement at the right shoulder joint. Ten months later she remained free from symptoms and further radiographs showed no recurrence of the lesion.

Case 2-An engineering fitter, aged thirty-nine years, complained of pain in the right shoulder region of five weeks' duration. He had received a blow on that shoulder one week before the onset of symptoms. On examination, there was a tender swelling over the medial part of the right clavicle. Movements of the right shoulder joint were full and painless.

Radiographs (Fig. 2) showed an area of destruction in the right clavicle adjacent to, but not involving, the sterno-clavicular joint. The inferior cortex at the site of the lesion was thinned and eroded, and there was some subperiosteal new bone formation at this point.

Investigations-Laboratory tests, including the differential white cell count and haematological examination of the bone marrow, were normal. 
A provisional diagnosis of multiple myelomatosis was not supported by further radiographic or by laboratory investigations, and so a biopsy was done.

Operation-The medial end of the right clavicle was exposed, and its cortex removed to reveal what

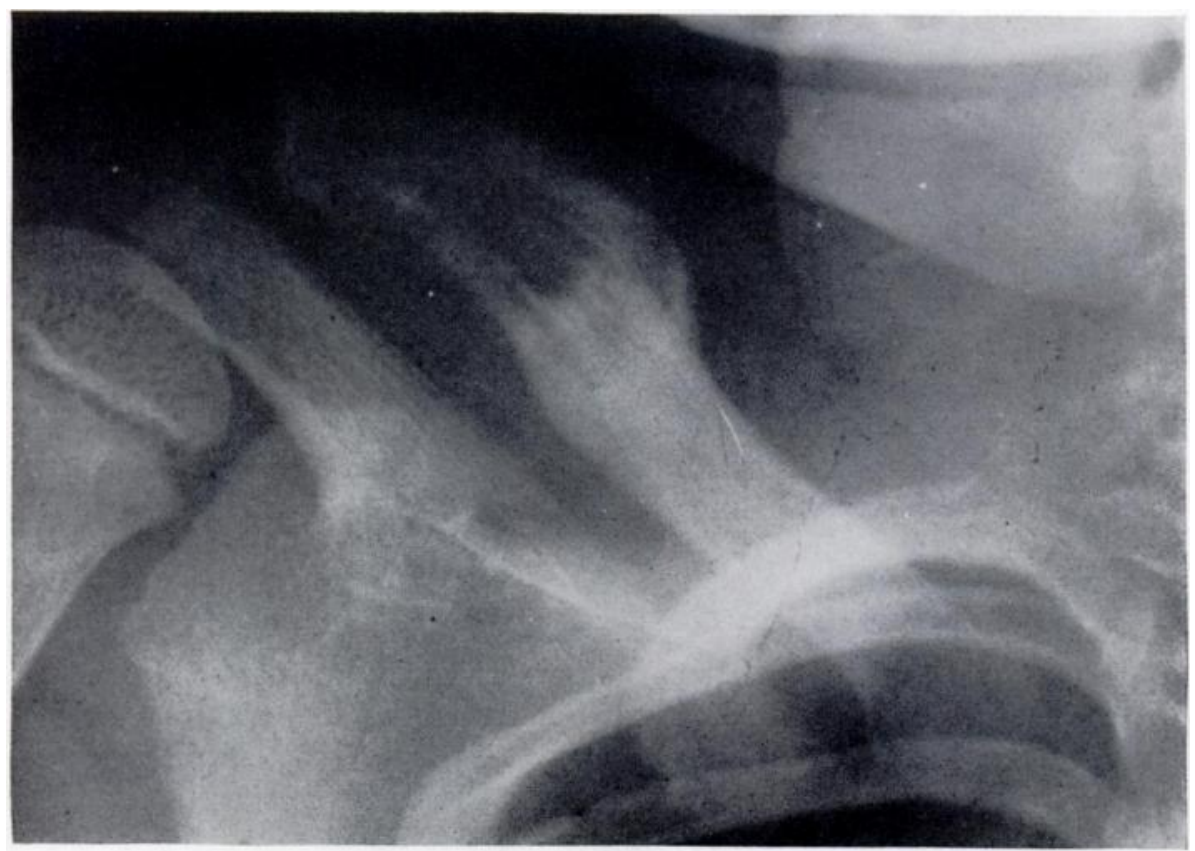

Fig. 1

Case 1-Showing destructive lesion of lateral half of clavicle, with subperiosteal new bone formation; fine crack fracture just visible.

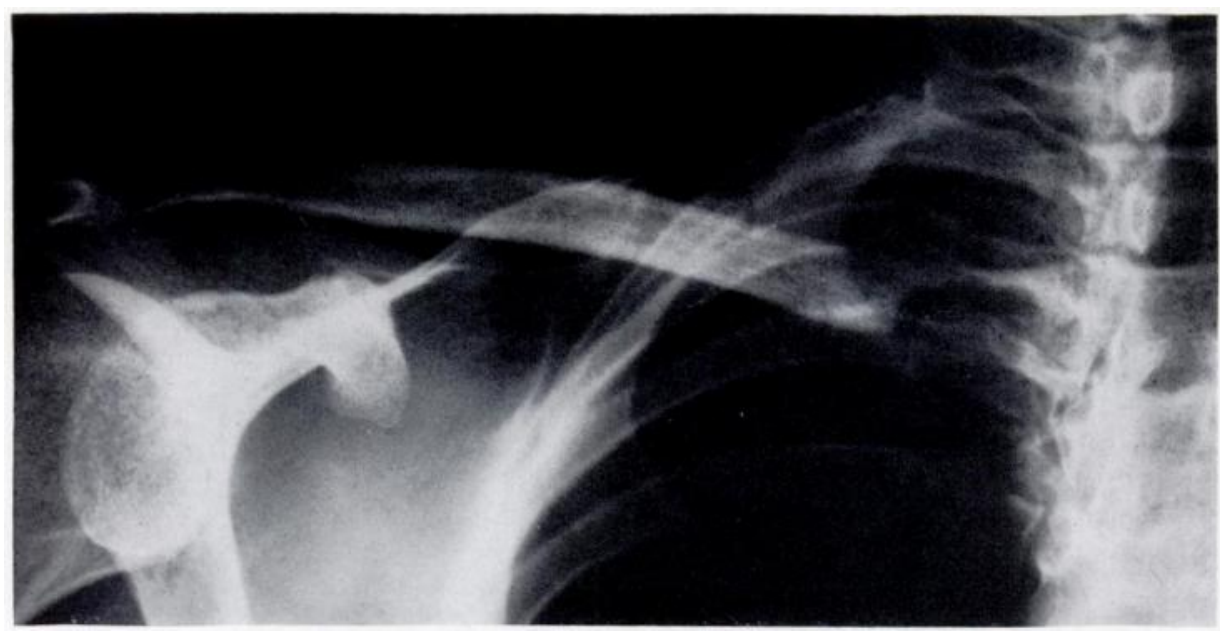

FIG. 2

Case 2-Showing destructive lesion at medial end of clavicle, with erosion of inferior cortex, and subperiosteal new bore formation.

appeared to be a soft, yellowish tumour mass. The medial third of the clavicle, including the whole of the diseased area, was excised.

Histology-The appearances were those of an eosinophilic granuloma.

Progress - The patient's post-operative course was uneventful. When examined one month later he was free from symptoms. As he then removed to another part of the country no detailed follow-up 
was possible, but his reply to a recent questionnaire indicates that, five years after the operation, his only complaints consist of slight weakness of the right shoulder and slight aching in that region, particularly when the upper limb is dependent for long periods.

Case 3-A motor mechanic, aged twenty-four years, had first noticed a lump on his left arm two weeks before attending hospital. The lump had enlarged rapidly and become increasingly painful. On examination, a firm, tender tumour three inches in diameter was found arising from the anterolateral aspect of the left humerus, about the level of the deltoid insertion. The overlying skin was not involved, and there was no adenopathy.

Radiographs (Fig. 3) showed a small area of destruction immediately under and involving the cortex of the left humerus. There was no evidence of periosteal reaction.

Investigations - Routine laboratory tests were normal.

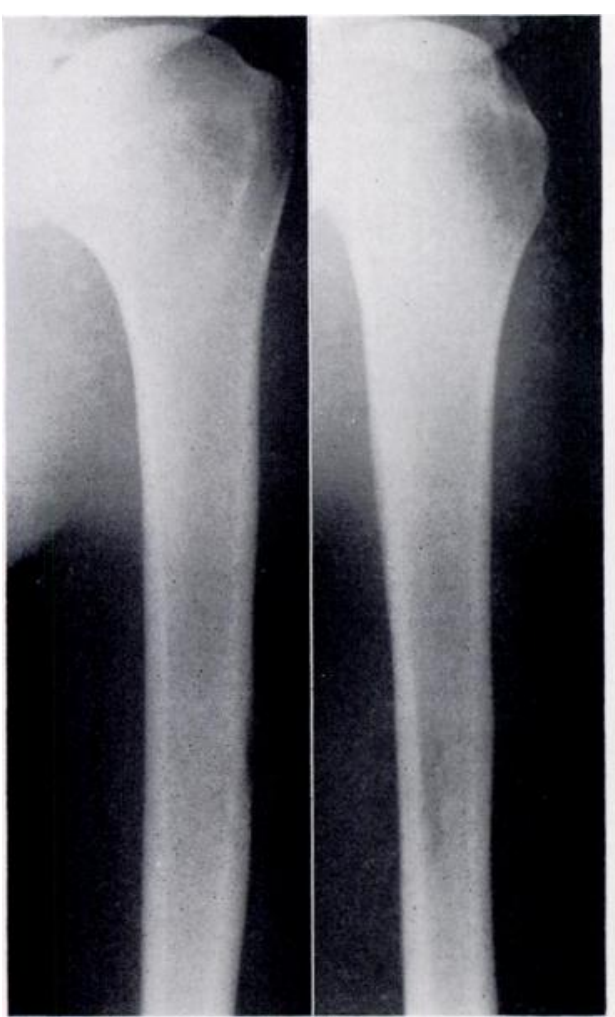

Fig. 3

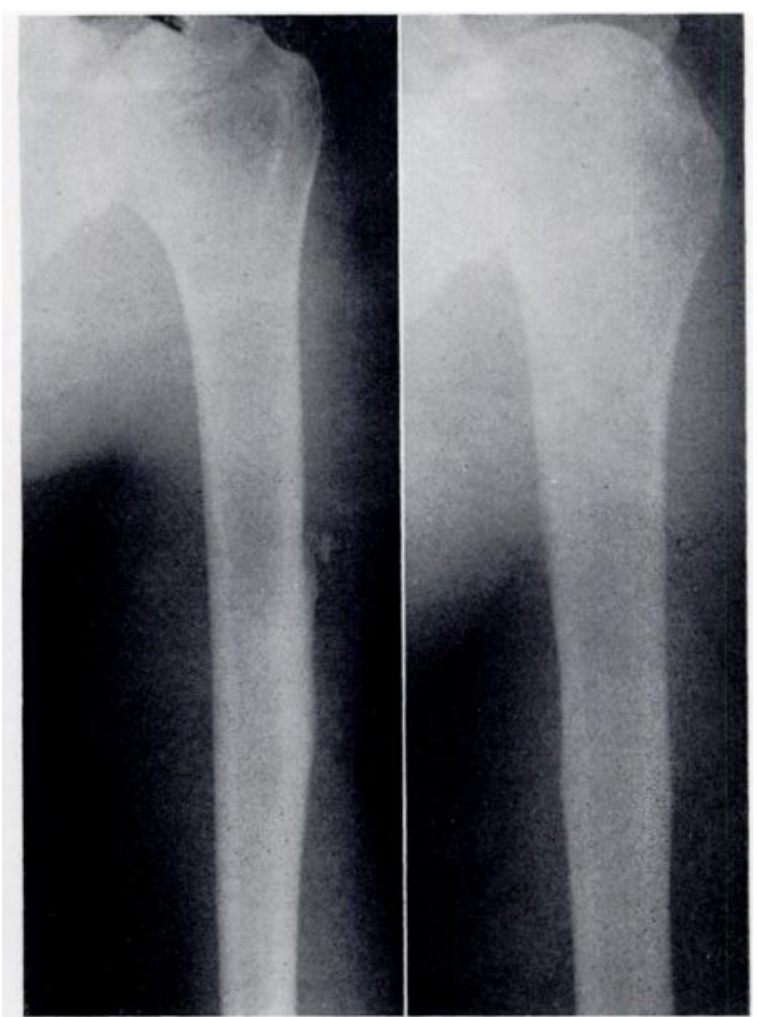

FIG. 4

Case 3. Figure 3-Small destructive lesion involving cortex of humerus. Figure 4-Seven months later, resolution complete, with slight residual thickening of cortex.

A biopsy was done. Although the radiographs were atypical of either condition, the rapid growth of the tumour suggested osteomyelitis or a malignant neoplasm.

Operation-The affected area of the left humeral shaft was exposed. The substance of the overlying brachialis muscle was found to be infiltrated by gritty material. The muscle and the periosteum were firmly adherent to the underlying bone. After the bone had been cleared, a defect, an inch in extent, was found in the cortex, through which a small amount of soft, yellow-brown material was removed with a curette.

Histology-The appearances of the curettings from the medullary cavity were considered to be those of an eosinophilic granuloma. A piece of the overlying muscle was also examined and found to be normal, apart from the presence of spicules of new bone formation.

Progress - The patient's pain was relieved after the biopsy operation. The swelling resolved, and when the arm was last examined seven months later the only clinical abnormality was a slight residual thickening of the humeral shaft. The radiographs at that time showed complete recalcification of the original defect, with slight residual thickening of the cortex, and a minor degree of ossification in the overlying soft tissue (Fig. 4).

VOL. 38 B, NO. 2, MAY 1956 
Case 4-A housewife, aged thirty-two years, complained of pain in her right lower ribs of six weeks' duration. On examination, there was tenderness accurately localised to one point on the tenth right rib.

Radiographs (Fig. 5) showed a small, irregular destructive lesion of this rib, with disappearance of a section of the cortical outline on its inferior border, and a little subperiosteal new bone formation.

A biopsy was done. The differential diagnosis lay between a secondary malignant deposit and an infective lesion.

Operation-The right tenth rib was exposed in the posterior axillary line, and the lesion identified. The cortex of the rib was found to have been penetrated by granulation tissue. A two-inch piece of the rib, containing the lesion, was excised.

Histology-The appearances were those of an eosinophilic granuloma.

Progress-The patient's post-operative course was uneventful. Serial radiographs over a follow-up period of nine months showed no recurrence of the rib lesion.

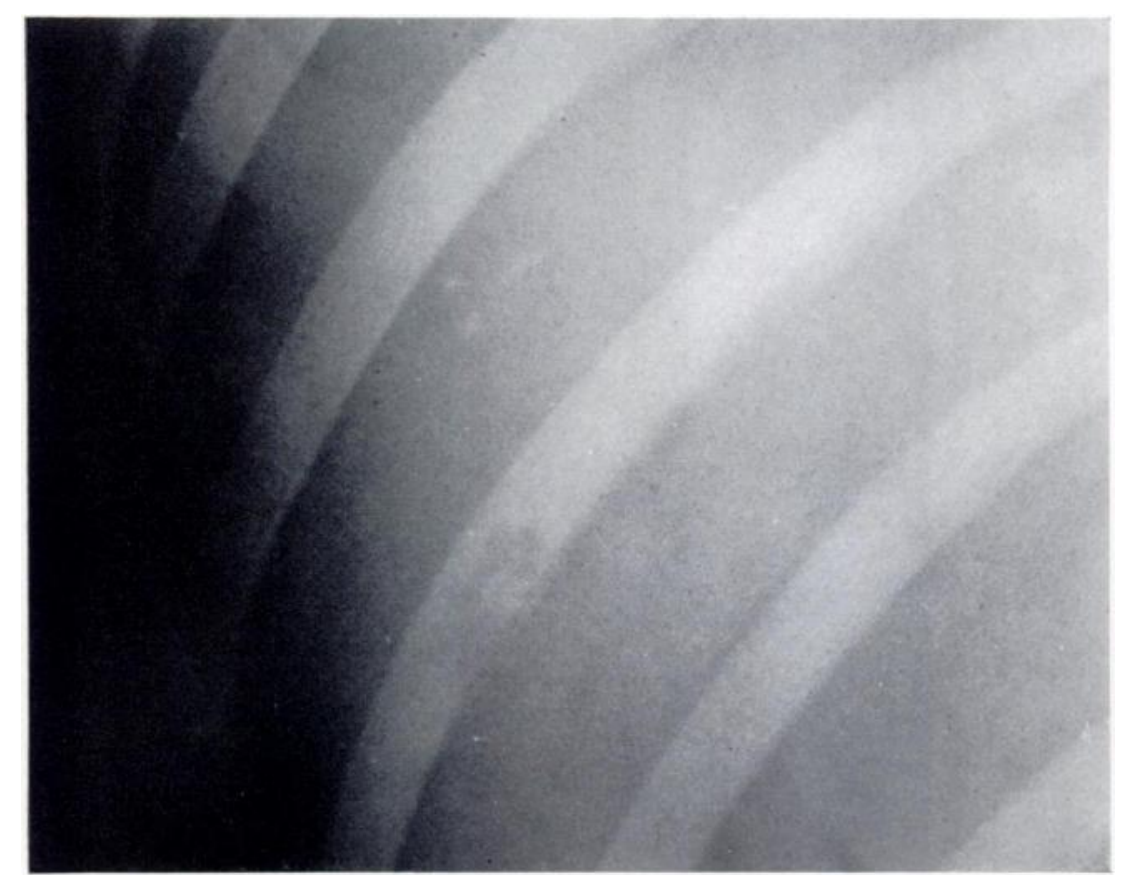

FIG. 5

Case 4-Showing destruction lesion of right tenth rib, with erosion of inferior cortex, and subperiosteal new bone formation.

Case 5-A boy, aged seven years, complained of pain in his left thigh of five weeks' duration, and had developed a limp. On examination, there was a slight flexion deformity of the left hip, and rotation movements were slightly limited by protective muscle spasm. The muscles of the left buttock and thigh showed slight wasting. The wing of the left ilium was tender on palpation.

Radiographs (Fig. 6) showed an irregular area of bone destruction in the left ilium, extending down to the acetabular margin. General osteoporosis of the upper end of the left femur was present, but the hip joint itself was not directly involved.

Investigations-Apart from a raised erythrocyte sedimentation rate (55 millimetres in the first hour) routine laboratory tests, including the differential white cell count, were normal.

A biopsy was done. The differential diagnosis lay between tuberculous osteitis and a primary or secondary neoplasm.

Operation-The outer table of the left ilium was exposed. It was found to be intact but soft and compressible over the lesion. A cortical window was removed immediately above the acetabulum, and a large cavity exposed containing gelatinous material. The cavity was cleared, and a specimen sent for histological examination and culture.

Histology-The appearances were those of an eosinophilic granuloma. Culture was sterile. 


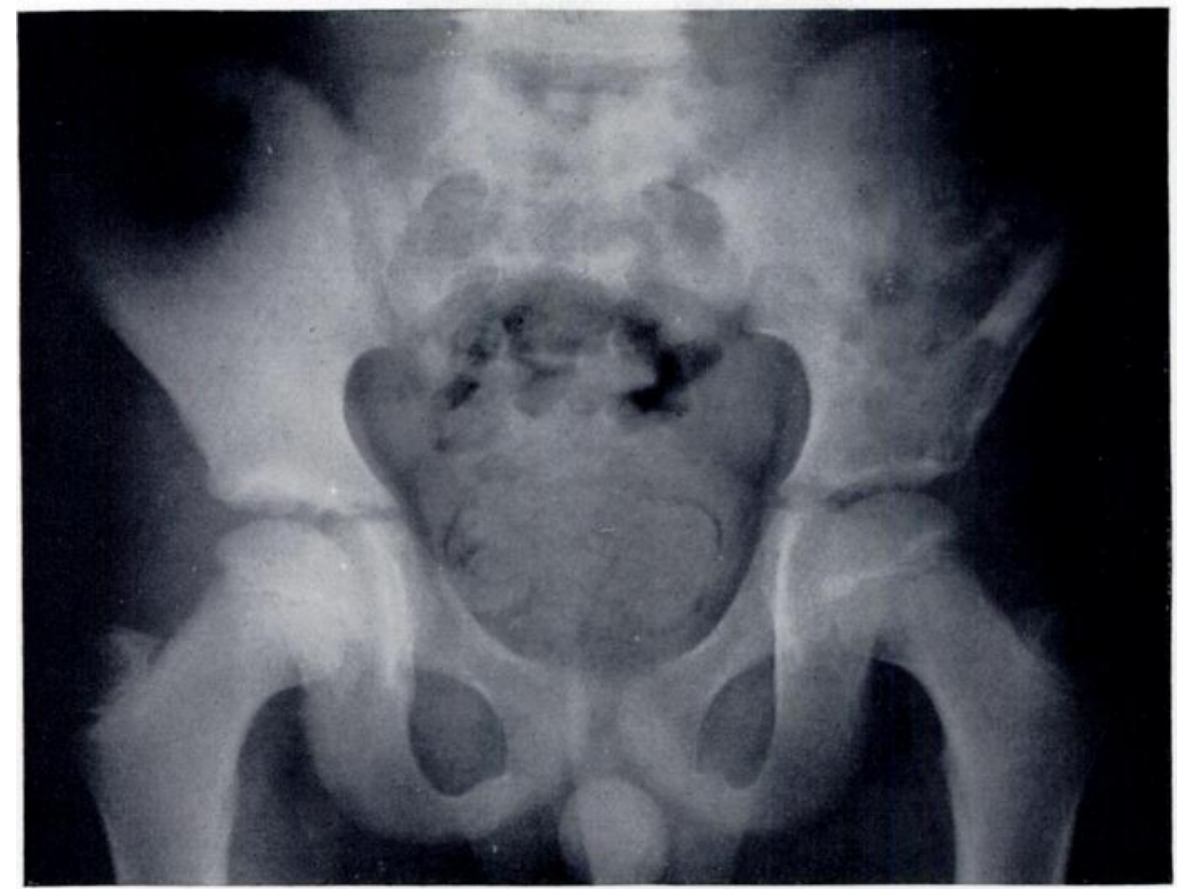

Fig. 6

Case 5-Showing irregular area of bone destruction in left ilium, with osteoporosis of upper end of femur.

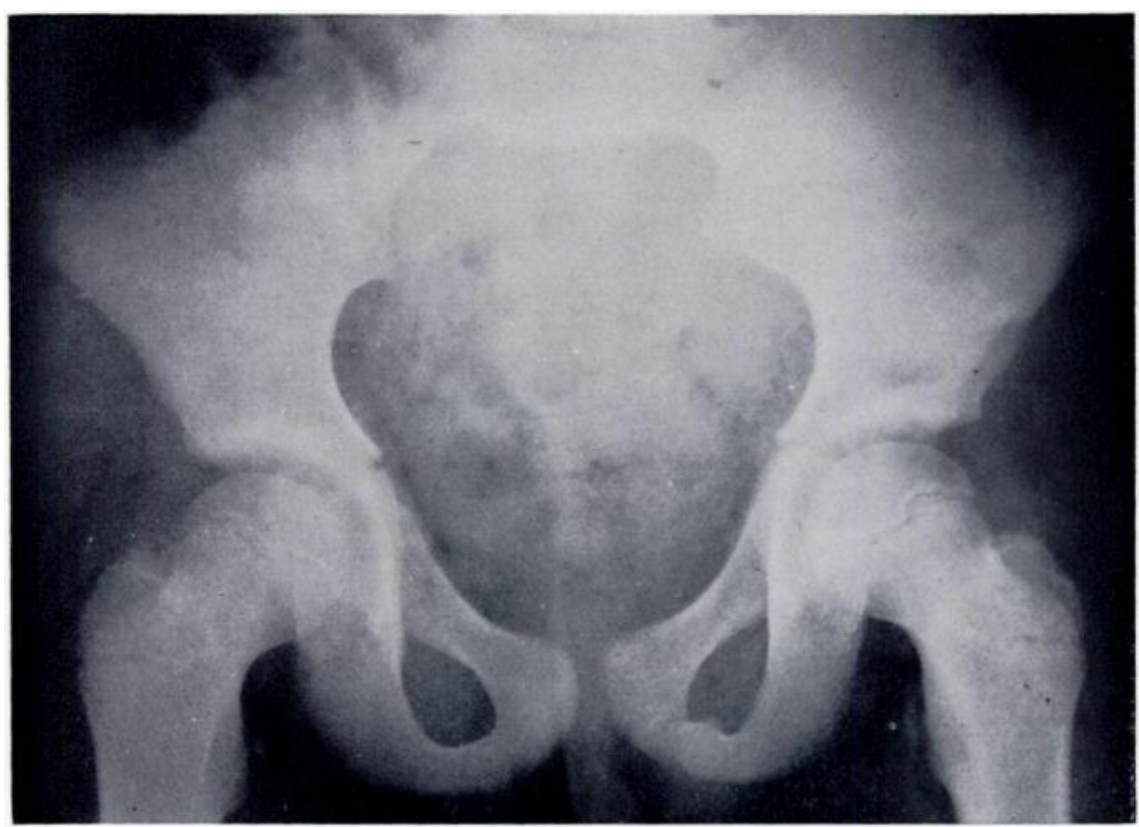

FIG. 7

Case 5-Two years later. The iliac lesion has healed, leaving only a small residual defect.

VOL. 38 B, NO. 2, MAY 1956 


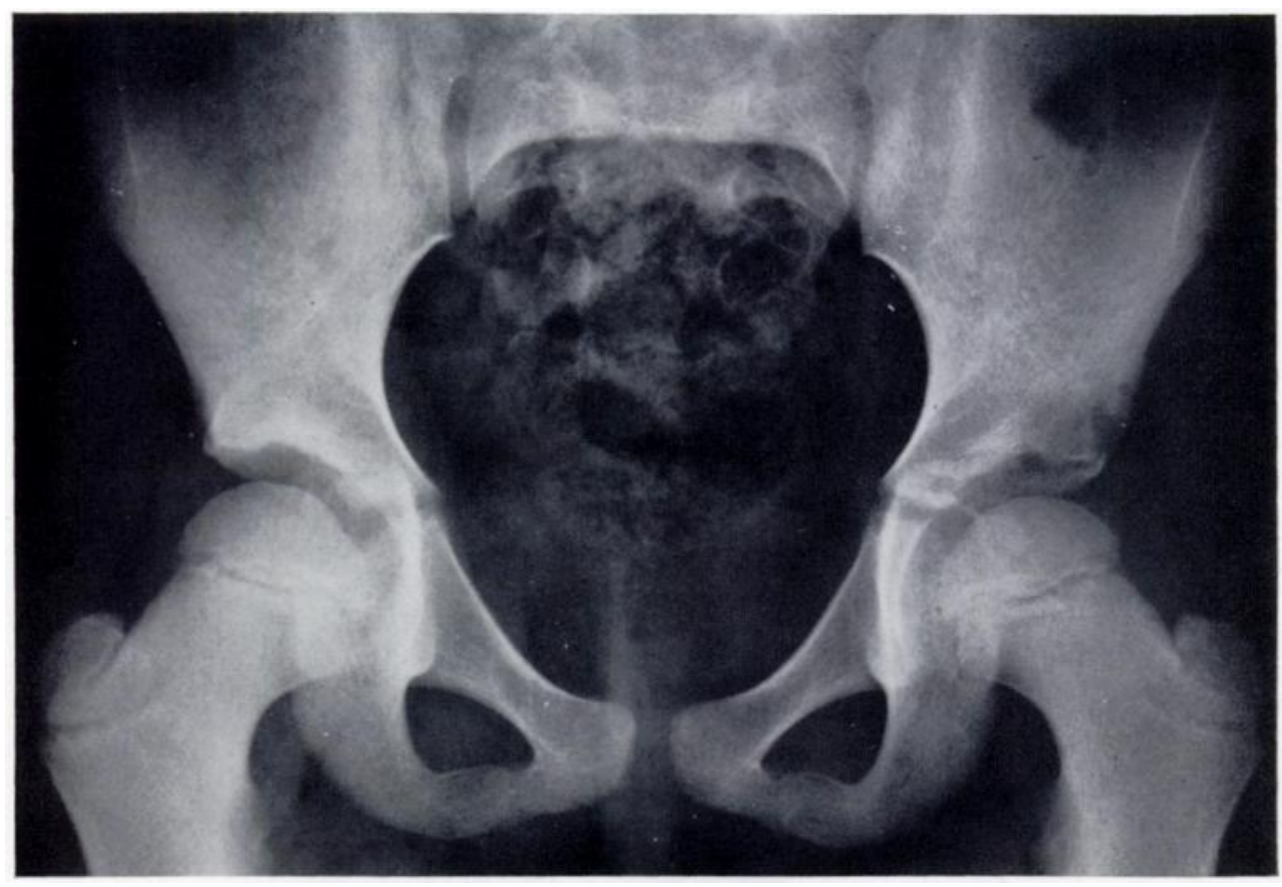

FIG. 8

Case 6-Showing destructive lesion in upper part of left acetabulum, with osteoporosis of bones around the hip joint.

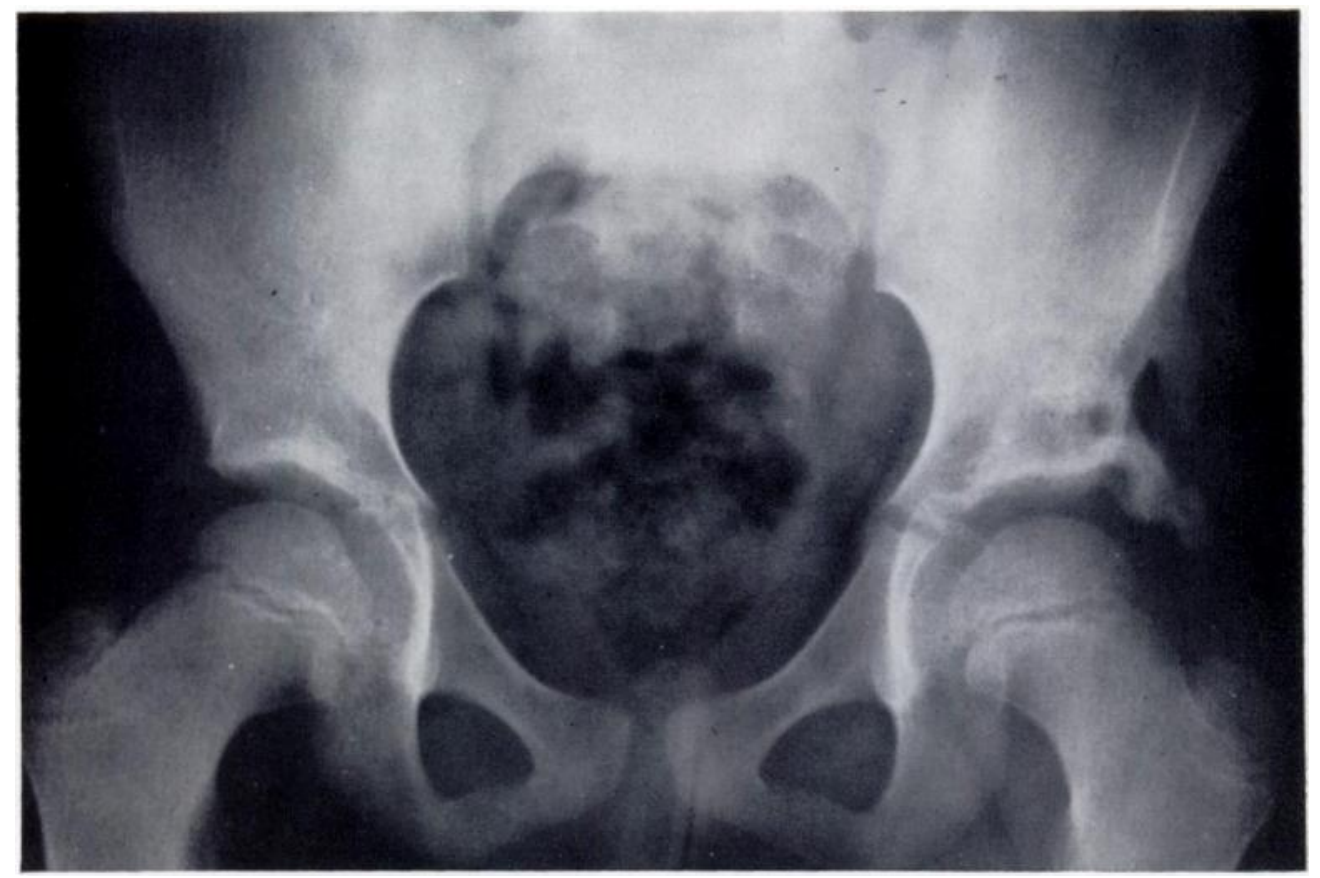

Fig. 9

Case 6-Fifteen weeks later, the lesion has recalcified, leaving a small defect above the acetabulum, and soft-tissue ossification. 
Progress-The post-operative course was uneventful. A course of deep x-ray therapy was given. The patient's pain was rapidly relieved and movements of the left hip returned to normal. Serial radiographs showed progressive recalcification of the iliac lesion. Two years later the patient remained free from symptoms, and at that time radiographs showed only a small residual defect above the left acetabulum (Fig. 7).

Case 6-A girl, aged six years, complained of pain in her left thigh of twelve days' duration, and had developed a limp two days before attending hospital. On examination, all movements of the left hip were slightly limited.

Radiographs (Fig. 8) showed a small area of bone destruction in the left ilium, related to the upper part of the acetabulum. There was some general osteoporosis of the bones around the left hip.

Investigations-Routine laboratory tests, including the differential white cell count, were normal.

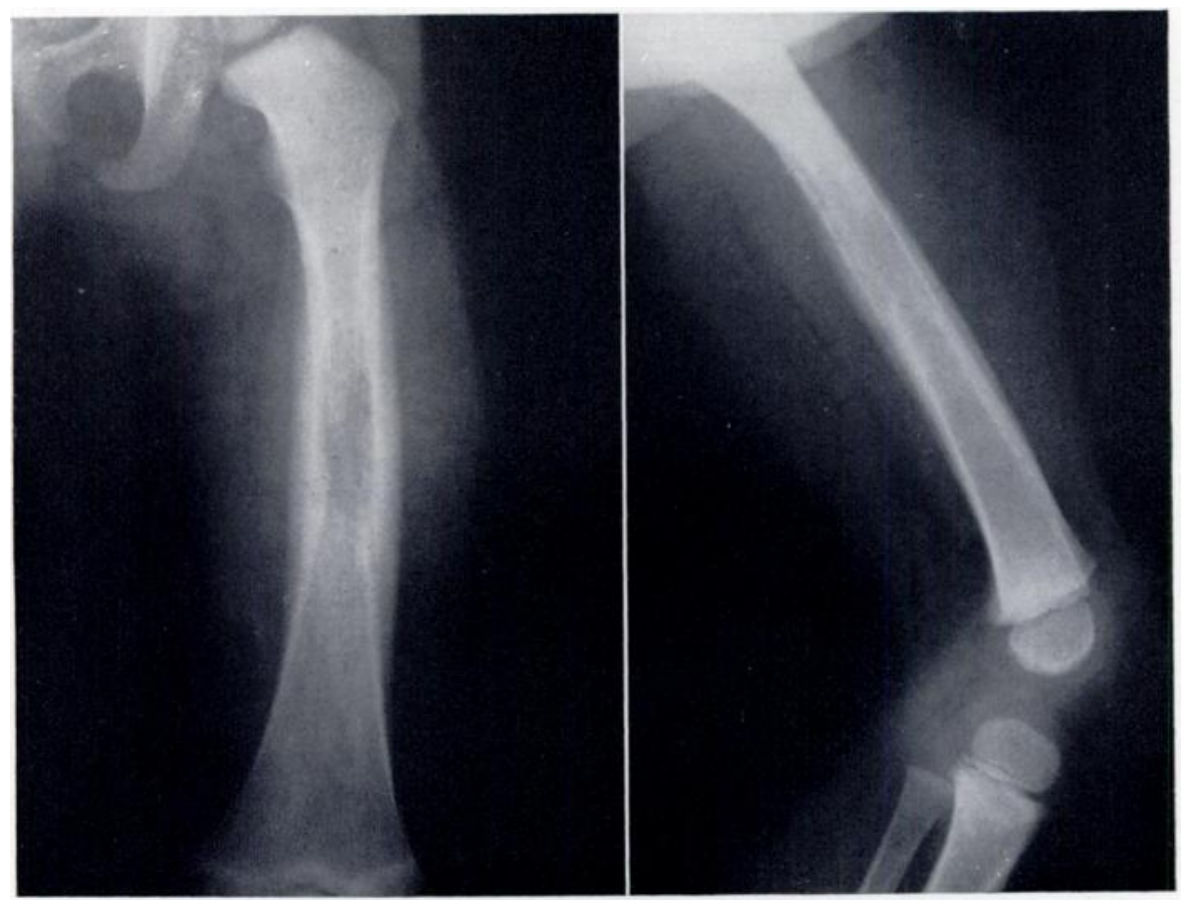

FIG. 10

Case 7-Destructive lesion in centre of femoral shaft, with surrounding subperiosteal new bone formation, laid down in "onion-skin" layers. hip joint.

A biopsy was done. The pre-operative diagnosis was that of a tuberculous infection of the left Operation-The superior aspect of the left hip was exposed through an anterior Smith-Petersen incision. A superficial cystic area which contained glairy fluid was found in the roof of the acetabulum, and deep to this was a cavity about an inch in diameter, containing reddish-grey vascular material. The cavity was curetted, and the incision closed. A specimen was sent for histological examination. Histology-The appearances were those of an eosinophilic granuloma.

Progress-After the biopsy operation skin traction was applied to the left lower limb for four weeks, and the patient was kept in bed for a further five weeks, before weight bearing was allowed. When last examined, fifteen weeks after operation, the child remained free from symptoms, movements of her left hip were normal and she walked without a limp. Radiographs then showed recalcification of the left iliac lesion with a small residual defect and two "stalactites " of soft-tissue ossification (Fig. 9).

Case 7-A girl, aged fifteen months, who had first walked at thirteen months, was observed by her parents to be limping. They attributed this to a fall one week prior to attending hospital. On examination, the only abnormal finding was a diffuse, slightly tender thickening of the left femoral shaft. 
Radiographs (Fig. 10) showed a destructive lesion in the centre of the left femoral shaft, surrounded by a considerable amount of subperiosteal new bone formation, laid down in "onion-skin " layers. Investigations-Routine laboratory tests, including erythrocyte sedimentation rate and differential white cell count, were normal.

A biopsy was done. A pre-operative diagnosis of Ewing's tumour was favoured, but the possibility of an eosinophilic granuloma was considered.

Operation-The affected part of the left femoral shaft was exposed. A cortical window was removed. exposing a cavity which contained yellowish gelatinous material. The cavity was cleared, and a specimen sent for histological examination and culture.

Histology-The appearances were those of an eosinophilic granuloma. Culture was sterile.

Progress-The left lower limb was not splinted. Eight days after the operation the child stood up in her cot and sustained a fracture through the diseased area of the femoral shaft (Fig. 11). The limb was immobilised on a Thomas's splint with fixed skin traction. The fracture united rapidly with

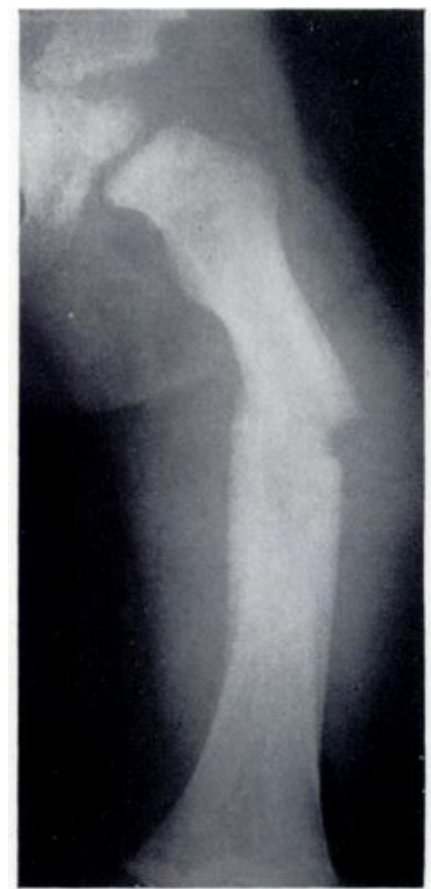

Fig. 11

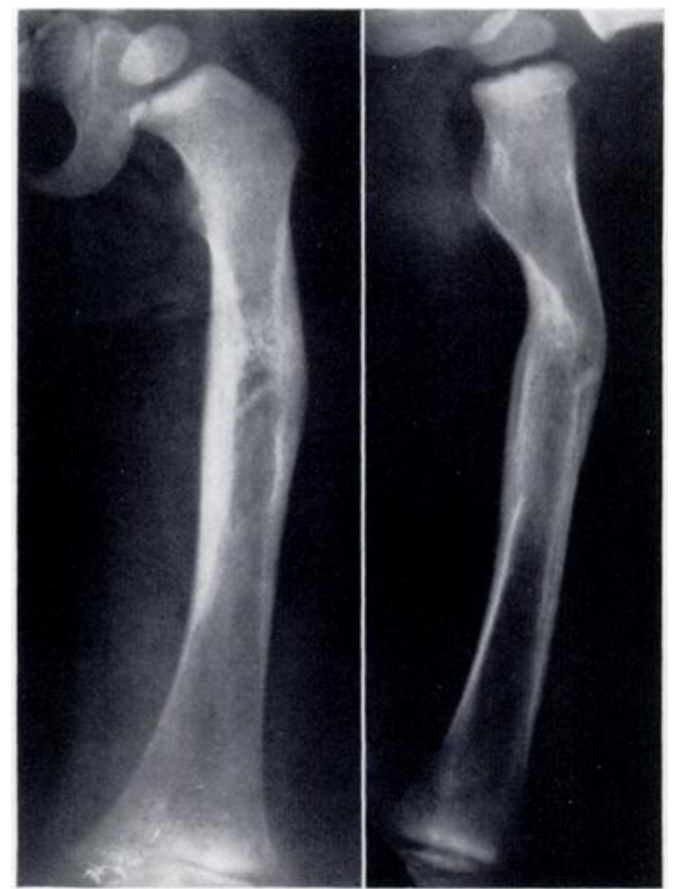

Fig. 12

Case 7. Figure 11-Fracture after biopsy operation. Figure 12-Six months later the fracture is soundly united. Partial recalcification of the original lesion has occurred, but an area of osteoporosis has appeared in the distal portion of the shaft.

abundant callus formation. Two months later weight bearing was allowed. When last reviewed, six months after the fracture, the child remained well and was walking normally. The radiographs (Fig. 12) showed sound union of the fracture; partial recalcification of the original lesion had occurred, but an area of osteoporosis was now present in the distal part of the shaft, requiring continued observation.

Case 8-A boy, aged nine years, who was a mental defective, complained of pain in his right axillary region of two months' duration. For three weeks before examination his parents had noticed increasing weakness of his right hand. On examination, there was profound weakness of the movements of the right hand and wrist. The right triceps jerk was absent. Owing to the patient's mental condition it was not possible to assess sensation. Examination of the remainder of the central nervous system was otherwise normal. There was a small cervico-thoracic kyphos, and forward flexion of the cervical spine was limited by pain and muscle spasm.

Radiographs (Fig. 13) showed a destructive lesion involving the right lamina and transverse process of the first thoracic vertebra. The lateral tomograph (Fig. 14) showed collapse of the body of the vertebra. 


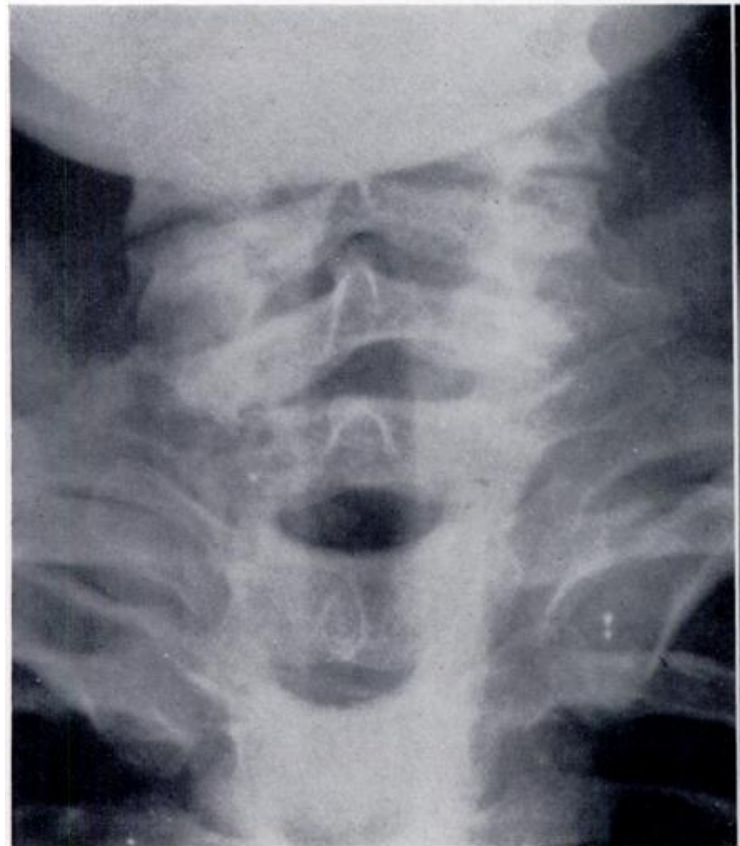

Fig. 13

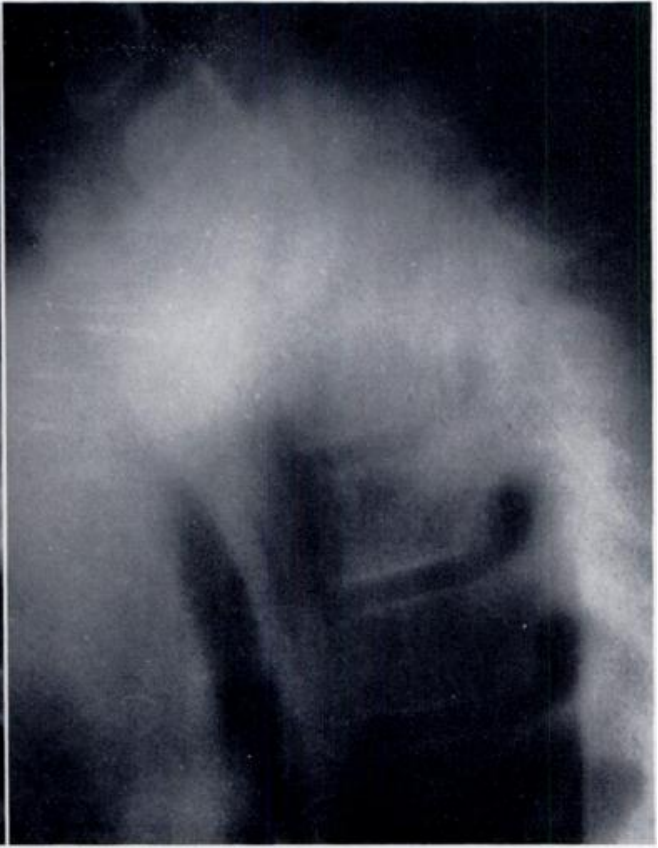

FIG. 14

Case 8. Figure 13-Showing destructive lesion of the right lamina and transverse process of first, thoracic vertebra. Figure 14-Lateral tomograph, showing collapse of the body of T.1.

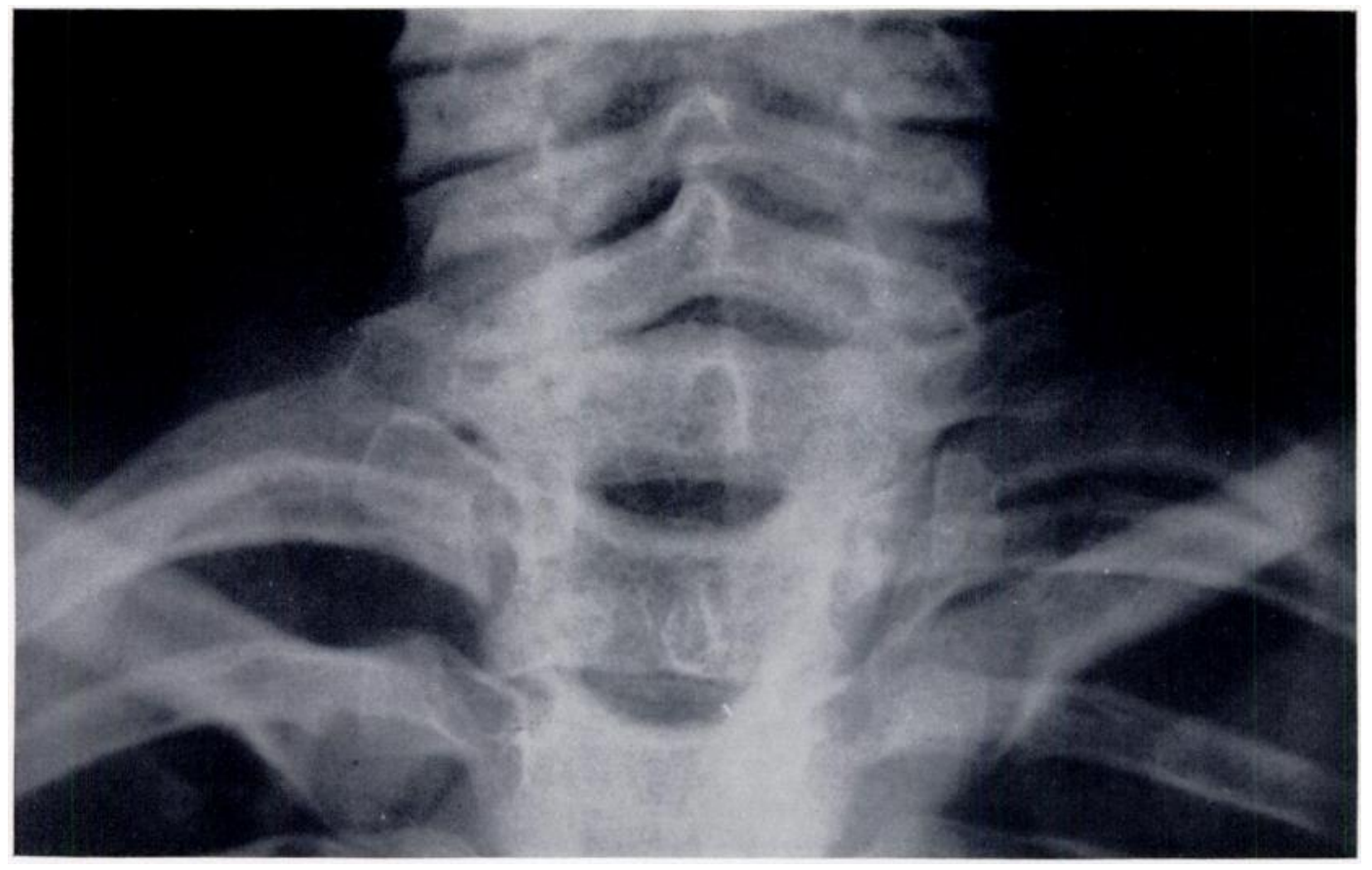

Fig. 15

Case 8-Eight months later, resolution is complete. 
Investigations-Lumbar puncture produced a slightly turbid yellow fluid containing: protein 150 milligrams per 100 millilitres; cells 3 red blood corpuscles per cubic millimetre, 2 lymphocytes per cubic millimetre. Culture was sterile. The remaining laboratory tests, including the white cell count, were normal.

A biopsy was done. The pre-operative diagnosis was that of a malignant neoplasm. Operation-The right lamina of the first thoracic vertebra was exposed, and was found to be partly invested by soft, rather gelatinous material which extruded through a small defect in the cortex. Part of this material was removed for histological examination, no attempt being made to explore the extent of the lesion.

Histology-The appearances were those of an eosinophilic granuloma.

Progress-A course of deep x-ray therapy was given, the patient being kept recumbent. The power of the right upper limb muscles improved rapidly. Two months after the biopsy no residual weakness remained, and he was allowed up, wearing a plastic collar, which was worn for a further three months. Serial radiographs showed recalcification of the vertebral lesion, progressing to complete resolution (Fig. 15). At the end of a two-year follow-up there has been no recurrence, and the patient remains free from symptoms.

\section{DISCUSSION}

The etiology of eosinophilic granuloma is unknown, but it is generally supposed to be a peculiar inflammatory reaction to some agent of infection, as yet unidentified. Cultures of biopsy material in these cases were sterile, except in Case 1, but the organisms isolated in this one instance were almost certainly contaminants.

The age incidence is in accordance with that quoted by previous writers; the condition is most frequently encountered in children and young adults. Although the sex incidence in this small series is equal, Dundon et al. (1946) have noted a preponderance in males, their estimated ratio being five males to one female.

Any bone proximal to the wrist and ankle may be the site of an eosinophilic granuloma, but the skull, ribs, femur, vertebrae, pelvis, mandible, humerus, and clavicles are most commonly affected. In the case of the long bones the lesion is usually, though not necessarily, situated in the shaft.

Pathology-The macroscopic appearances are those of a brownish, yellow material of the consistency of porridge, occupying the medullary cavity. The cortex of the affected bone may be thinned, eroded through, or thickened by reactive subperiosteal new bone formation. The overlying periosteum is closely adherent to the bone, or it may be perforated.

Histologically seven of these cases presented the main features of eosinophilic granuloma which, as summarised by Jaffe and Lichtenstein (1944), include: 1) Sheet-like formations of histiocytes ("the basic component "). 2) More or less conspicuous numbers of eosinophilic cells, especially of eosinophilic leucocytes. 3) Actively phagocytic multinuclear giant cells. The photomicrograph in Case 7 (Fig. 16) illustrates these appearances.

In Case 3, eosinophilic cells were a less conspicuous feature, but their presence in focal collections against a background of fibroblasts and pale-staining cells with abundant vacuolated cytoplasm was sufficiently diagnostic to place the case in the eosinophilic granuloma group.

Jaffe and Lichtenstein demonstrated that complete and spontaneous resolution can occur directly from the stage described above, but Green and Farber (1942) suggested that resolution might also occur through a subsequent and intermediate stage, in which the histological picture changes to that of a lipogranuloma, reminiscent of a Hand-Schüller-Christian lesion. In the latter, large vacuolated mononuclear cells predominate, eosinophils disappear, and the ingrowth of connective tissue is evident. The microscopic appearances in Case 3, indeed, suggest such an intermediate stage.

Clinical features-Eosinophilic granuloma may be a completely silent lesion. In three of the eight cases here described, symptoms were first noticed after an injury.

In none of the cases was there any significant systemic disturbance. 
The presenting symptoms and signs of the solitary lesion are generally local pain and swelling in the case of the long bones. The severity of the pain varies, but is not usually considerable. In other sites attention may be first drawn to the condition by involvement of a related structure.

Laboratory investigations-Routine laboratory investigations do not help diagnosis, being normal in most cases.

Occasionally leucocytosis is encountered (as in Case 1), and the erythrocyte sedimentation rate may be raised (as in Case 5). Eosinophilia (4 to 11 per cent) has been recorded as an occasional finding (Dundon et al. 1946) but was not found in the present cases. Several writers have noted an increased serum alkaline phosphatase, as found in Case 1.

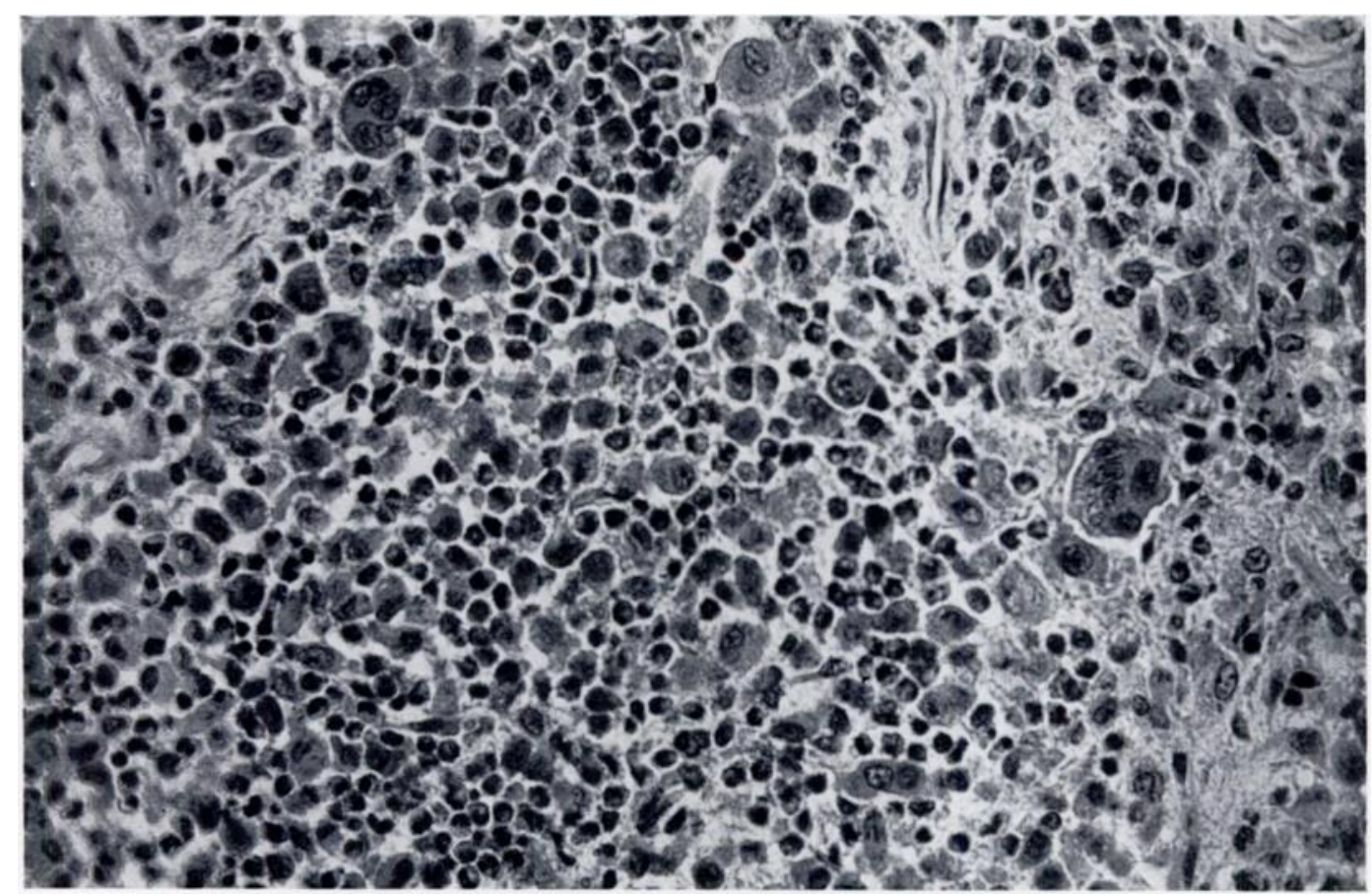

FIG. 16

Photomicrograph in Case 7, showing basic pattern of large mononuclear cells, interspersed with large numbers of eosinophilic leucocytes, and several multinuclear giant cells. (Haematoxylin and eosin, $\times 400$.)

The cerebrospinal fluid in Case 8 showed a raised protein content, but no increase in its cellular constituents. This resulted presumably from the obstruction of cerebrospinal fluid pathways.

In Hill's (1949) case it was possible to establish a diagnosis by aspiration of the fluctuant swelling over a skull lesion: the cellular constituents of the serous fluid withdrawn contained 56 per cent of eosinophils.

Radiographic features-The lesion presents as an area of endosteal destruction, irregular in outline, or with a smooth contour producing a "punched out" effect. As it enlarges it eventually erodes the cortex, a section of which may completely or partly disappear.

Subperiosteal new bone formation is regarded by most authors as unusual. When it occurs it is said to be associated with perforation of the cortex (Fairbank 1951). Four of the present cases showed subperiosteal new bone formation. In Cases 1 and 7 it was quite a marked feature (Figs. 1 and 10). The radiographs in Case 7 in particular show well defined "onion-skin" layers resembling the classical description of Ewing's tumour. Cortical 
perforation into the overlying soft tissues was observed at operation in only one of these cases, but all four showed radiographic evidence of complete destruction of parts of the original cortex.

The lesion is said to cause cortical expansion in some cases, and it may also appear loculated (Hill 1949), but neither of these features was evident in the cases here described.

The radiographic appearances of eosinophilic granuloma may be confused with a variety of other conditions, including pyogenic, syphilitic and tuberculous osteomyelitis, HandSchüller-Christian disease, osteitis fibrosa cystica, osteoclastoma, Ewing's tumour, osteogenic sarcoma, multiple myelomatosis, and metastatic tumours.

Complications-Pathological fractures are uncommon. Hill (1949) found only four cases in his review of the literature. The fracture occurring in Case 7 (Fig. 11) does not entirely merit such a description as it occurred soon after a biopsy operation, during which part of the femoral cortex had been removed. The rapidity with which union subsequently occurred, however, is noteworthy (Fig. 8).

The radiographs in Case 1 (Fig. 1) showed a crack fracture through the site of the clavicular lesion. This no doubt produced the first symptom of pain, and so in this instance explains the relationship of trauma to the onset of symptoms.

The involvement of the nerve roots in Case 8, with profound motor weakness, is of interest chiefly because of the rapidity with which complete recovery of the paresis occurred after radiotherapy to the first thoracic vertebra. That the latter was extensively involved is demonstrated by the radiographs (Figs. 13 and 14), which showed collapse of the body in addition to destructive changes in the right lamina and transverse process. In view of the rapid disappearance of the paresis it seems likely that the actual mode of involvement of the nerve roots was by local pressure and oedema associated with the presence of granulation tissue in the extradural space.

Green and Farber (1942) described a case of eosinophilic granuloma of a vertebra in which radiographs showed a related spindle-shaped soft-tissue shadow, resembling a cold abscess. This resolved completely without neurological complications.

Treatment and prognosis-There is no doubt from observations made by several writers on proved cases of eosinophilic granuloma, in which multiple foci were present, that complete and spontaneous recovery of the lesion can and does occur, with restitution of the normal radiographic appearances of the involved bone.

In view of the lack of specific diagnostic criteria short of biopsy, however, it follows that all cases of solitary eosinophilic granuloma of bone, once demonstrated radiographically, are likely to be explored. To curettage is then attributed the subsequent healing of the lesion. Diagnostic considerations and healing apart, curettage appears to fulfil an additional function in rapidly relieving the patient's symptom of pain, probably by decompression of the lesion. This was particularly noticeable in Case 3 , in which the pain, being relatively acute, disappeared immediately after operation.

Since resolution may occur either spontaneously or after curettage, the place of radiotherapy in the treatment of eosinophilic granuloma is difficult to assess. It was employed originally as an empirical measure, and although most writers agree that it is successful in relieving pain, there is some doubt as to its actual effect on the pathological process. Hamilton et al. (1946) stated that they could find no radiographic evidence that radiotherapy altered the course of the disease. Hill (1949) concluded that the prognosis as regards spontaneous healing in the course of months was so good that it was unnecessary to employ radiotherapy as a routine measure. Dundon et al. (1946), however, described an eosinophilic granuloma of the sternum with an associated soft-tissue mass in the mediastinum. This lesion failed to settle after diagnostic curettage, and on three occasions recurring symptoms were relieved by radiotherapy. On each occasion the mediastinal mass, as seen in the radiographs, was observed to diminish in size after treatment. 
It seems reasonable, therefore, in the solitary eosinophilic granuloma, to employ radiotherapy, not as a routine measure, but for the few cases which fail to resolve after curettage. It may also be used as an additional safeguard in cases in which rapid resolution is particularly desirable because of involvement or imminent involvement of important related structures such as nerve roots.

As already indicated, the prognosis of the solitary lesion is excellent, its benign course apparently being maintained even in the presence of such complications as those described. In the presence of multiple lesions it is agreed that the general outlook is also good, although in view of the relationship established with the more sinister conditions of Hand-SchüllerChristian disease and Letterer-Siwe disease, a more guarded prognosis may be advisable. A radiographic survey of the skeleton is, therefore, of some clinical importance when the apparently solitary lesion presents itself.

\section{SUMMARY}

1. Eight cases of solitary eosinophilic granuloma of bone are described, along with two possible complications.

2. The main pathological, clinical, and radiographic features of the condition, and its treatment, are discussed.

3. The essentially benign course of the solitary lesion, even in the presence of complications, is confirmed.

I am indebted to Mr F. W. Holdsworth, Mr R. A. King, Mr L. C. Oliver, Mr N. Roberts and Mr D. Wainwright for permission to publish cases under their care; to Dr E. A. Atkinson and Dr I. M. Larkin for their aid with the histological examination; to Dr J. M. Stewart for his advice and for interpreting the radiographs; and to $\mathrm{Mr} \mathrm{H}$. Osmond-Clarke for criticism and encouragement. My thanks are also due to Mr E. A. Sheppard of the Photographic Department, Oldchurch Hospital, for preparing the radiographic prints, and to Mr E. V. Wilmott for the photomicrograph.

\section{REFERENCES}

Dundon, C. C., Williams, H. A., and LaipPly, T. C. (1946): Eosinophilic Granuloma of Bone. Radiology, 47, 433.

FaIrbank, Sir T. (1951): Eosinophilic Granuloma of Bone. In Atlas of General Affections of the Skeleton, p. 300. Edinburgh and London: E. \& S. Livingstone Ltd.

Green, W. T., and Farber, S. (1942): " Eosinophilic or Solitary Granuloma " of Bone. Journal of Bone and Joint Surgery, 24, 499.

Hamilton, J. B., Barner, J. L., Kennedy, P. C., and McCort, J. J. (1946): The Osseous Manifestations of Eosinophilic Granuloma. Radiology, 47, 445.

HILl, R. M. (1949): Non-Specific (Eosinophilic) Granuloma of Bone. British Journal of Surgery, 37, 69.

Jaffe, H. L., and Lichtenstein, L. (1944:) Eosinophilic Granuloma of Bone. Archives of Pathology, $37,99$. Lichtenstein, L., and JAFFe, H. L. (1940): Eosinophilic Granuloma of Bone, with Report of Case. American Journal of Pathology, 16, 595.

Otani, S., and Ehrlich, J. C. (1940): Solitary Granuloma of Bone, Simulating Primary Neoplasm. American Journal of Pathology, 16, 479.

Ponsetr, I. (1948): Bone Lesions in Eosinophilic Granuloma, Hand-Schüller-Christian Disease, and LettererSiwe Disease. Journal of Bone and Joint Surgery, 30-A, 811. 